\title{
Ipsilateral Double Parathyroid Adenoma and Thyroid Hemiagenesis
}

\author{
W.K. Mydlarz ${ }^{\text {a }} \quad$ K. Zhang ${ }^{\text {a }} \quad$ S.T. Micchellib ${ }^{b} \quad$ M. Kim ${ }^{\text {c }} \quad$ R.P. Tufano ${ }^{a}$ \\ Departments of a Otolaryngology - Head and Neck Surgery, ${ }^{b}$ Pathology, and c Division of Endocrinology and \\ Metabolism, Johns Hopkins University School of Medicine, Baltimore, Md., USA
}

\section{Key Words}

Thyroid dysgenesis $\cdot$ Hemiagenesis $\cdot$ Double parathyroid adenoma $\cdot$ Hyperparathyroidism

\begin{abstract}
Background/Aims: To describe a case of left thyroid dysgenesis, accompanied by ipsilateral double parathyroid adenomas in a setting of primary hyperparathyroidism, and to review the pertinent literature on the diagnosis of these rare clinical scenarios. Methods: Review of the English literature with addition of a case report. Results: Preoperative evaluation included both sestamibi and ultrasound evaluation of the neck. Fine-needle aspiration biopsies of what was thought to be two concerning thyroid nodules revealed potential double intrathyroidal parathyroid adenomas. Videoassisted exploration verified double parathyroid adenomas and revealed concomitant left thyroid lobe dysgenesis. Intact parathyroid hormone level returned to normal and a greater than $50 \%$ drop from baseline was achieved intraoperatively with subsequent long-term cure. Conclusions: Thyroid dysgenesis is a rare, poorly understood and potentially confusing variety of developmental anomalies, which can be associated with thyroid as well as parathyroid disease. Clinical diagnosis is highly dependent upon the clinician maintaining an index of suspicion for these anomalies, thorough physical examination and careful review of available imaging modalities, especially while investigating thyroid and parathyroid disorders.

Copyright $\odot 2010$ S. Karger AG, Basel
\end{abstract}

\section{Introduction}

Hemiagenesis is a rare but benign congenital anomaly in which one of the thyroid lobes fails to develop. The cause of hemiagenesis is not clear, but it is believed that the defect may arise from failure of the embryological development of a thyroid lobe [1]. We recently encountered a rare case of left thyroid dysgenesis, accompanied by ipsilateral double parathyroid adenomas in a patient with primary hyperparathyroidism. Institutional review board approval was obtained.

\section{Case Report}

A 55-year-old woman with primary hyperparathyroidism was referred to Johns Hopkins Hospital for surgical evaluation. She presented with new-onset constipation and dyspepsia, and several years of fatigue, hip pain, memory loss, reflux, decreased concentration as well as depression. Laboratory evaluation revealed a total serum calcium of $12.8 \mathrm{mg} / \mathrm{dl}$ (normal $8.4-10.5 \mathrm{mg} / \mathrm{dl}$ ) with an elevated intact parathyroid hormone (PTH) level of $478 \mathrm{pg} / \mathrm{ml}$ (normal $10-65 \mathrm{pg} / \mathrm{ml}$ ). The total 25 -hydroxy vitamin D was 26 $\mathrm{ng} / \mathrm{ml}$, and thyroid function tests were normal. A dual energy Xray absorptiometry scan showed evidence of osteoporosis with a total radius T score of -3.6. She denied any history of head or neck irradiation, thyroid problems, pancreatitis, nephrolithiasis, and any family history of multiple endocrine neoplasia syndromes.

Primary hyperparathyroidism was suspected and the patient underwent sestamibi single photon emission CT imaging. Early images demonstrated asymmetric thyroid uptake of radiotracer left greater than right, with retention of radiotracer in what was

\section{KARGER}

Fax +41613061234 E-Mail karger@karger.ch www.karger.com
Dr. Ralph P. Tufano

Department of Otolaryngology - Head and Neck Surgery

601 N. Caroline Street, JHOC 6210

Baltimore, MD 21287 (USA)

Tel. +1 410955 3628, Fax +1 410955 0035, E-Mail rtufano@ jhmi.edu 
Fig. 1. Early (a) and 2-hour delayed (b) left anterior oblique views on sestamibi single photon emission CT imaging demonstrating asymmetric thyroid uptake of radiotracer (left greater than right), with 2 discrete foci of radiotracer uptake thougth to be in the left thyroid lobe.

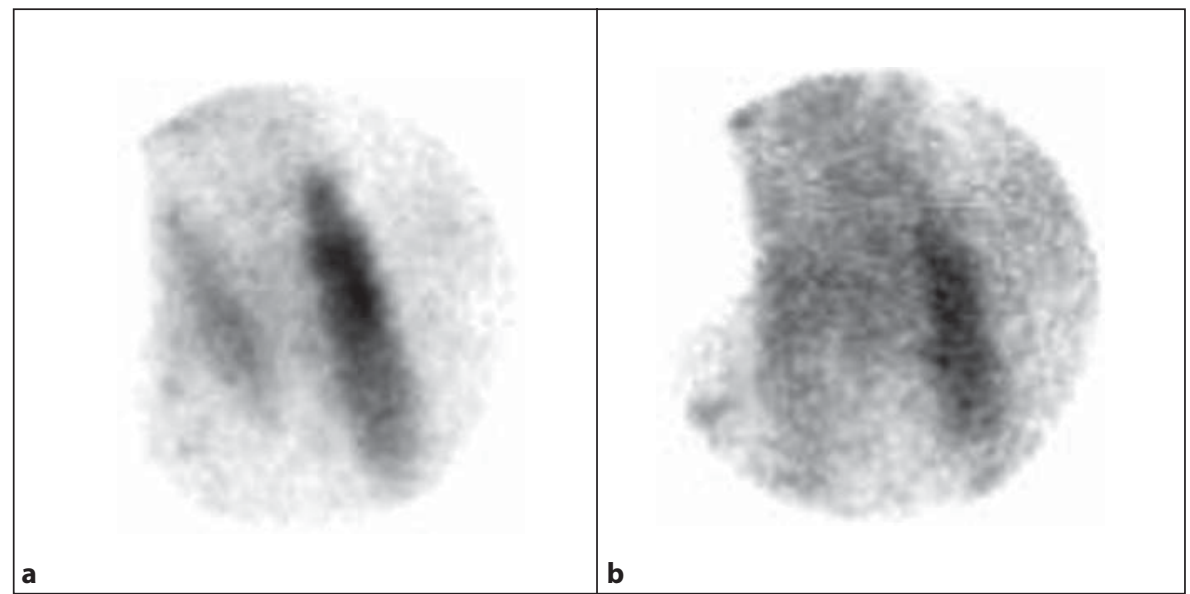

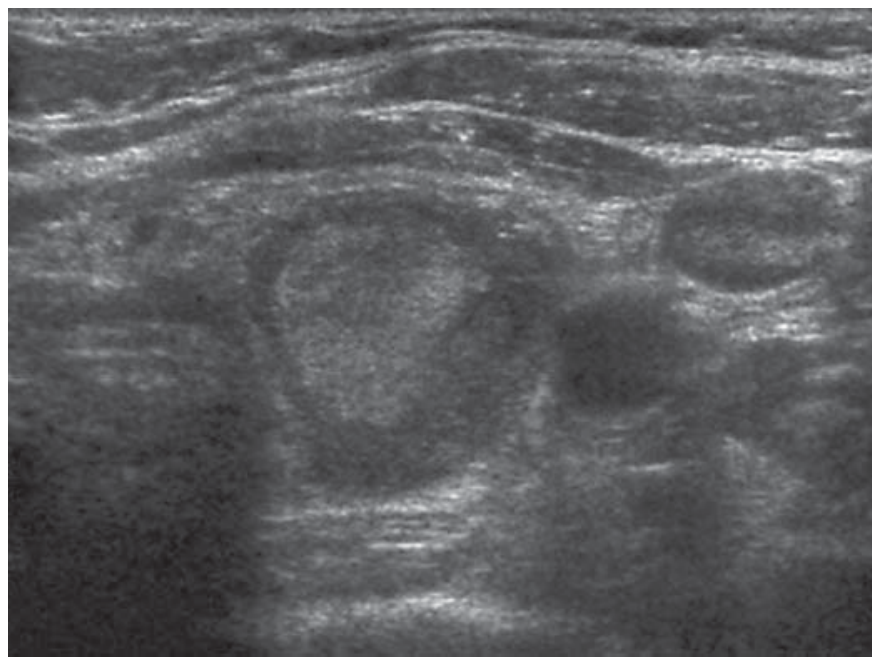

Fig. 2. Thyroid ultrasound demonstrating what was thought to be a left inferior thyroid nodule measuring $1.7 \mathrm{~cm}$ in its largest dimension. The left common carotid artery and internal jugular vein are located laterally and the trachea medially to this suspected thyroid nodule.

thought to be the left thyroid lobe and washout of radiotracer from the right on the delayed images (fig. 1). This seemed most consistent with an intrathyroidal left-sided parathyroid adenoma. Thyroid ultrasound seemingly demonstrated a multi-nodular goiter with the right and left lobe measuring $5.7 \times 1.4 \times 2.0 \mathrm{~cm}$ and $6.2 \times 1.3 \times 1.6 \mathrm{~cm}$, respectively, with two left-sided 1.7 - and 1.4-cm nodules (fig. 2). An ultrasound-guided fine-needle aspiration biopsy was performed to rule out malignancy. Both aspirates had elevated PTH levels and cytopathology was consistent with parathyroid tissue.

Under the diagnosis of primary hyperparathyroidism, left neck exploration was performed. Two masses were removed with-

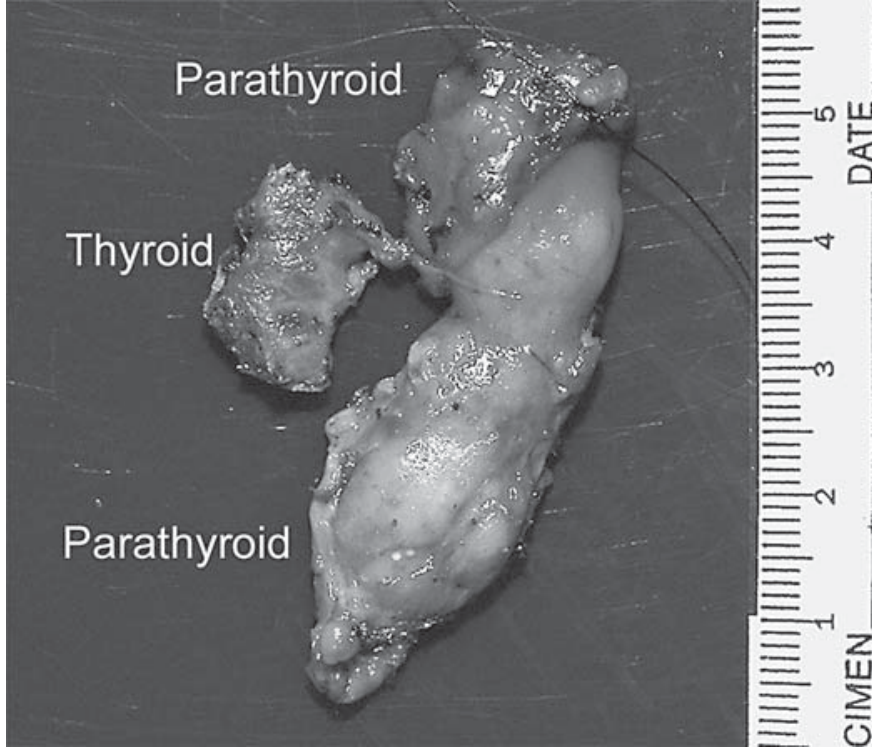

Fig. 3. Gross surgical picture of the left thyroid lobectomy and parathyroidectomy specimen revealing 2 adjacent parathyroid gland adenomas with a markedly atretic left thyroid lobe, attached to the superior parathyroid gland adenoma by a small pedicle.

out any discernable thyroid tissue was appreciated. Surgical pathology showed 2 left-sided parathyroid adenomas measuring 2.2 and $2.4 \mathrm{~cm}$, superiorly and inferiorly. There was only a small fragment of histologically unremarkable thyroid tissue (fig. 3). The preoperative intact PTH was $434 \mathrm{pg} / \mathrm{ml}$, and 5, 10, and $20 \mathrm{~min}$ after excision intact PTH levels were 38,38 and $43 \mathrm{pg} / \mathrm{ml}$, respectively. The patient was discharged on postoperative day 1 without any signs of hypocalcemia and a total serum calcium of $9.2 \mathrm{mg} /$ $\mathrm{dl}$, and remained eucalcemic without any evidence of secondary hyperparathyroidism at the 12-month follow-up visit. 


\section{Discussion}

Thyroid dysgenesis is a rare and potentially confusing anomaly, especially in the setting of double parathyroid adenomas and primary hyperparathyroidism. This clinical scenario presents an interesting dilemma in the characterization of the specific type of thyroid dysgenesis in this patient as the final pathology revealed only a tiny amount of histologically unremarkable thyroid tissue. This case more closely resembles hemiagenesis rather than thyroid hypoplasia, as the patient was euthyroid and had a normally functioning right thyroid lobe [1]

The overall prevalence and incidence of thyroid hemiagenesis varies among reports, but are assumed to be about 0.02 and $0.01 \%$, respectively $[2,3]$. Thyroid hemiagenesis is associated with a 3:1 female predominance, and the left lobe of the thyroid is absent far more often than the right lobe, at a ratio of 4:1, similarly to our case [4]. Previous studies that defined hemiagenesis relied on radiographic imaging and not surgical exploration and tissue analysis $[3,5]$. Conventional radiographic imaging has resolution detection limitations, and it is difficult to verify thyroid hemiagenesis without surgery. Hence, our case can be better characterized as near complete thyroid hemiagenesis, if not effective thyroid hemiagenesis.

The clinical diagnosis of thyroid hemiagenesis is highly dependent upon the clinician maintaining an index of suspicion. The physical examination in our patient demonstrated normal size and consistency of the thyroid, with the right lobe slightly larger than the left, making the diagnosis difficult just based on the physical examination due to the presence of significant ipsilateral double parathyroid adenomas. In this scenario, ultrasoundguided fine-needle aspiration and analysis of intact PTH in the aspirate proved helpful to distinguish these questionable 'thyroid nodules' as parathyroid disease, but near complete thyroid hemiagenesis could not be confirmed until surgical intervention. This case highlights the difficulties of diagnosing thyroid hemiagenesis and is the first case report of near thyroid hemiagenesis and ipsilateral double parathyroid adenomas.

\section{References}

1 De Felice M, Di Lauro R: Thyroid development and its disorders: genetics and molecular mechanisms. Endocr Rev 2004;25:722746.

- 2 Harada T, Nishikawa Y, Ito K: Aplasia of one thyroid lobe. Am J Surg 1972;124:617-619.

-3 Shabana W, Delange F, Freson M, Osteaux M, De Schepper J: Prevalence of thyroid hemiagenesis: ultrasound screening in normal children. Eur J Pediatr 2000;159:456458 .
4 Melnick JC, Stemkowski PE: Thyroid hemiagenesis (hockey stick sign): a review of the world literature and a report of four cases. J Clin Endocrinol Metab 1981;52:247-251.

5 Maiorana R, Carta A, Floriddia G, Leonardi D, Buscema M, Sava L, Calaciura F, Vigneri $\mathrm{R}$ : Thyroid hemiagenesis: prevalence in normal children and effect on thyroid function. J Clin Endocrinol Metab 2003;88:1534-1536. 